\section{Vorzeichenwechsel bei der Diffusionswärme}

Von Ludwig Waldmann

Aus dem Kaiser-Wilhelm-Institut für Chemie, Tailfingen (Z. Naturforschg. 2a, 358 [1947]; eingegangen am 4. Juni 1947)

Zur Untersuchung der Temperaturabhängigkeit des Thermodiffusionsfaktors, welcher zugleich auch für die Diffusionswärme maßgebend ist, sind die auf letzterer beruhenden Meßmethoden besonders geeignet, weil dabei fast einheitliche Temperatur herrscht und weil das Verfahren so sehr einfach ist ${ }^{1}$. Anläßlich diesbezüglicher Versuche konnte nun festgestellt werden, daß bei der Temperatur des flüssigen Sauerstoffs die Diffusionswärme von $\mathrm{N}_{2} / \mathrm{A}$ und von $\mathrm{O}_{2} / \mathrm{A}$ das entgegengesetzte Vorzeichen hat wie bei Zimmertemperatur. Diese Beobachtung fügt sich neueren Erfahrungen bei der Thermodiffusion von $\mathrm{NH}_{3} / \mathrm{Ne}^{2}$ und ${ }^{14} \mathrm{NH}_{3} /{ }^{15} \mathrm{NH}_{3}{ }^{3}$ an.

Zwecks Vermeidung mechanisch $\mathrm{zu}$ bewegender Apparaturteile wurde der stationäre Diffusionsthermoeffekt herangezogen und ein Strömungsdoppelrohr nach dem früher angegebenen Schema benutzt ${ }^{1}$. Das ebenfalls schon früher beschriebene Verfahren, welches auf der Messung des exakt berechenbaren Temperaturlinienintegrals beruhte, konnte allerdings nicht angewandt werden, da die hierbei zulässigen Strömungsgeschwindigkeiten der Gase und demgemäß auch die zu beobachtenden Temperaturunterschiede zu klein sind. Die Strömungsgeschwindigkeiten wurden vielmehr so groß gewählt - und für beide Gase gleich -, daß die Temperaturunterschiede infolge der Diffusionswärme extremal sind. Wie man aus den Grundgleichungen des Diffusionsthermoeffekts entnimmt, gilt für die extremale Temperaturdifferenz, gemittelt längs der Rohrachse, die Beziehung

$$
\left(T-T_{0}\right)_{\text {extrem }}=T_{0} \frac{\mathfrak{R}}{C_{p}} a\left(\gamma_{\infty}-\gamma_{-\infty}\right) \bar{\Theta}_{\max }\left(\frac{K}{D}\right) \text {. }
$$

Darin bedeuten $T_{\mathbf{0}}$ die Temperatur der Rohrwand, $\Re$ die Gaskonstante, $C_{p}$ die Molwärme, $\alpha$ den Thermodiffusionsfaktor, $\gamma_{-\infty}$ bzw. $\gamma_{\infty}$ den Molenbruch der einen Komponente beim Einströmen bzw. nach völliger Vermischung. $\bar{\Theta}_{\max }$ ist ein durch Eichung zu ermittelnder Zahlenfaktor, welcher bei gegebener Geometrie des Apparats nur noch rom Verhältnis $K / D$ des Temperaturleit- zum Diffusionskoeffizienten abhängt.

Der benutzte Strömungsapparat bestand aus zwei etwa $4 \mathrm{~cm}$ langen Rohren ron je $0,75 \mathrm{~cm}$ Durchmesser, welche sich in einem $0,4 \mathrm{~cm}$ breiten, netzbedeckten Schlitz berührten und in deren Achsen je ein $8 \mu$ Golddraht, der als Widerstandsthermometer diente, gespannt war. Zur Ermittlung von dessen Temperaturempfindlichkeit wurde die bekannte Dampfdruckkurve des flüssigen Sauerstoffs herangezogen. Die Badtemperatur war kurzdauernd auf weniger als $0,001^{\circ}$ konstant. Die Gase wurden ror dem Eintritt in den Strömungsapparat in Kupferschlangen auf die Badtemperatur abgekühlt. In allen aufgeführten Fällen war $K / D \approx 1$. Aus einer Messung des $\left(\overline{\left.T-T_{0}\right)}\right.$ extrem von $\mathrm{N}_{2} / \mathrm{A}$ bei Zimmertemperatur ergab sich mit der Annahme $\alpha=0,071$ der Wert $\bar{\Theta}_{\max }(1)=0,10_{6}$, welcher sodann für die Auswertung der anderen Messungen zugrundegelegt wurde.

\begin{tabular}{|c|c|c|c|c|}
\hline & \multicolumn{2}{|c|}{$293^{\circ} \mathrm{K}$} & \multicolumn{2}{|c|}{$89^{\circ} \mathrm{K}$} \\
\hline & $\begin{array}{c}T-T_{0} \text { extrem } \\
\text { in }\end{array}$ & $a$ & $\begin{array}{c}\left|\overline{T-T_{0}}\right| \text { extrem } \\
\text { in }{ }^{0}\end{array}$ & $a$ \\
\hline $\mathrm{N}_{2} / \mathrm{A}$ & 0,37 & $0,071^{*}$ & 0,035 & $-0,022$ \\
\hline $\mathrm{O}_{2} / \mathrm{A}$ & 0,26 & 0,051 & 0,053 & $-0,034$ \\
\hline $\mathrm{N}_{2} / \mathrm{O}_{2}$ & 0,077 & 0,018 & $<0,0015$ & $\mid \alpha<0,001$ \\
\hline
\end{tabular}

* Annahme; s. Text.

Tab. 1. Gemessene Werte der extremalen mittleren Temperaturdifferenz $\left|T-T_{0}\right|_{\text {extrem längs der Achse }}$ und die daraus entnommenen Thermodiffusionsfaktoren $\alpha$.

Die Versuchsergebnisse sind aus Tab. 1 zu ersehen. Die Temperaturänderungen infolge der Diffusionswärme waren in allen Fällen in beiden Rohren entgegengesetzt und im Absolutbetrag höchstens um etwa $30 \%$ verschieden. Angegeben sind mittlere Werte. Positives Vorzeichen von $\alpha$ bedeutet, daß sich die schwere Komponente abkühlt (und dementsprechend im Temperaturgefälle die kalte Gegend bevorzugen wird). Bei $\mathrm{N}_{2} / \mathrm{O}_{2}$ und $89^{\circ} \mathrm{K}$ war keinerlei Diffusionswärme zu beobachten. Störungen durch Kondensation von $\mathrm{O}_{2}$ traten nicht auf.

Die Vorzeichenumkehr des Thermodiffusionsfaktors ist ein besonders deutlicher Ausdruck für das „Weicherwerden" der Moleküle bei abnehmender Temperatur.

1 Siehe den zusammenfass. Bericht L. Waldmann, Z. Naturforschg. 1, 59 [1946].

2 K. E. Grew, Nature [London] 150, 320 [1942].

3 W. W. Watson u. D. Wo ernley, Physic. Rev. 63, 181 [1943].

\section{Das Bandenspektrum und die Dissoziations- wärme des $\mathrm{BrF}$}

Von Peter Heinz Brodersen und Hans Joachim Schumacher ${ }^{1}$

(7. Naturforschg. 2a, 358-359 [1947]; eingeg. am 2. April 1947)

Das Spektrum des BrF wurde mit einem $Z$ ei $\beta$ schen Drei-Prismen-Spektrographen, Kamera $f=87 \mathrm{~cm}$, Dispersion etwa $12 \AA / \mathrm{mm}$, aufgenommen. Als Re-

1 Diese und die fünf folgenden Arbeiten wurden in den Jahren 1938 bis 1944 im Institut f. physik. Chemie der Univ. Frankfurt a. M. fertiggestellt. 